\title{
Die Struktur klimaskeptischer Argumente
}

\section{Verschwörungstheorie als Wissenschaftskritik}

Klimaskeptiker(innen) stellen sich gegen den Mainstream und behaupten, dass der wissenschaftliche Konsens über die Ursachen der globalen Erwärmung nicht nur falsch, sondern gefälscht sei. Sie gehen von einer Verschwörung der Klimaforscher(innen) aus. Die folgende Analyse klimaskeptischer Argumentation beruht auf einer Jens Soentgen, Helena Bilandzic Auswertung deutsch- und englischsprachiger Sachbücher. Sie legt Funktion und Begründung der klimaskeptischen Emotionalisierungsstrategie offen.

The Structure of Climate Skeptical Arguments. Conspiracy Theory as a Critique of Science GAIA 23/1 (2014): 40-47

\begin{abstract}
Questioning and rejecting the scientific consensus on climate change causes, climate skeptics argue that climate science is involved in a global conspiracy, guided by self-interest and aspirations of societal change. On the basis of 97 climate skeptical non-fiction books, this article reconstructs the logical structure of climate skeptical (or denialist) arguments, with a special focus on the construction of the causes for climate change. The analysis shows that climate skeptical arguments are made on three levels: on the level of insight from climate science, on the level of an intuitive sociology of science and on a political/economic level, thus achieving the desired effect of moving the audience emotionally. The reconstruction of arguments may serve as a basis for more quantitative research, but it is also useful for entering the debate with climate skeptics as it allows anticipating climate skeptical objections.
\end{abstract}

Keywords

climate science, climate skeptics, $\mathrm{CO}_{2}$, denialism, environmental communication, global warming, sociology of science

Kontakt: Dr. Jens Soentgen | Universität Augsburg | Wissenschaftszentrum Umwelt (WZU) | Universitätsstr. 1a | 86159 Augsburg | Deutschland | Tel.: +498215983560 | E-Mail: soentgen@wzu.uni-augsburg.de

Prof. Dr. Helena Bilandzic | Universität Augsburg | Institut für Medien, Wissen und Kommunikation | Augsburg | E-Mail: helena.bilandzic@phil.uni-augsburg.de

(C) 2014 J. Soentgen, H. Bilandzic; licensee oekom verlag. This is an Open Access article distributed under the terms of the Creative Commons Attribution License (http://creativecommons.org/licenses/by/3.0), which permits unrestricted use, distribution, and reproduction in any medium, provided the original work is properly cited.

\section{Das Phänomen Klimaskepsis}

Klimaskepsis - die Ablehnung zentraler Annahmen der Klimaforschung - ist nicht nur, aber zu einem großen Teil ein wissenschaftsexternes Phänomen: Sie wird in vielen populärwissenschaftlichen und -kulturellen Büchern vertreten (Crichton 2005, Vahrenholt und Lüning 2012) und reflektiert (Hoggan 2009, Hulme 2009) sowie in Massenmedien (Antilla 2005, Gavin und Marshall 2011, Jacques et al. 2008, Poortinga et al. 2011, Whitmarsh 2011) und auf Webseiten ${ }^{1}$ verbreitet. Am klimaskeptischen Diskurs beteiligen sich nicht nur Journalist(inn)en und Einzelautor(inn)en, sondern auch konservative Thinktanks liefern einen wesentlichen Beitrag (Jacques et al. 2008). Klimaforscher(innen) sehen solche Tendenzen mit Besorgnis (zum Beispiel Nicholson 2010, Weart 2011); einige der Argumente werden von der Klimaforschung ihrerseits in Blogs und anderen Publikationen geprüft und widerlegt. Die klimaskeptischen Diskurse werden (fach)wissenschaftsintern diskutiert (zum Beispiel Kerr 2009, Nicholson 2010, Weart 2011), aber eher punktuell (etwa als Reaktion auf Studien oder Leserbriefe) und nicht systematisch aufgearbeitet. Ziel dieses Beitrags ist es, das Phänomen Klimaskepsis näher zu bestimmen und die Struktur der Argumentation anhand eines umfangreichen Korpus an deutsch- und englischsprachigen klimaskeptischen Sachbüchern nachzuvollziehen.

\section{Forschung zum klimaskeptischen Diskurs}

In der bisherigen Forschung zum Phänomen Klimaskepsis (zum differenzierten Gebrauch siehe unten) wurden zum einen prominent die institutionellen Zusammenhänge zwischen politischen und kommerziellen Interessen und dem klimaskeptischen Diskurs beleuchtet. Oreskes und Conway (2010) analysieren in einer politikwissenschaftlich gearbeiteten, akteurszentrierten Studie,

1 Zum Beispiel www.climate-skeptic.com. 
welche politischen und personellen Verknüpfungen unter einer kleinen Auswahl US-amerikanischer klimaskeptischer Personen existieren. Sie kommen dabei zum Ergebnis, dass diese in politischen Thinktanks organisiert sind und oft finanzielle Unterstützung durch politisch motivierte Organisationen oder industrienahe Stiftungen erhalten, um ihre klimaskeptischen Ansichten zu verbreiten - daher auch die von dem Autorenteam gewählte Bezeichnung merchants of doubt. Der klimaskeptische Diskurs an sich wurde jedoch nicht betrachtet.

Im Gegensatz dazu beschäftigen sich auch einige Studien mit dem Diskurs selbst. So vermutet Leuschner (2012, S. 2) in ihrer wissenschafts- und erkenntnistheoretischen Analyse der Glaubwürdigkeit von Wissenschaft, vor allem der Klimaforschung: „Forschung wird genau dann zum Gegenstand öffentlicher Glaubwürdigkeitsdebatten, wenn epistemische Unsicherheiten auf politische, moralische, soziale oder ökonomische Interessen treffen". Aus wissenschaftstheoretischen Thesen wie der von der Theoriebeladenheit der Beobachtung leitet sie mögliche klimaskeptische Einwände ab (Leuschner 2012, S. 93-99) und diskutiert diese. Auf die im Diskurs konkret geäußerten skeptischen Argumente, die sich von wissenschaftsphilosophischen Konstrukten in der Regel beträchtlich unterscheiden, geht sie jedoch nur mit sehr wenigen Beispielen ein, die ausschließlich deutsche Publikationen einbeziehen (Leuschner 2012, S. 106). Leuschners Vermutung, dass im klimaskeptischen Diskurs auch „politische, moralische, soziale und ökonomische Interessen“ artikuliert werden, wird gleichwohl von unserer Analyse bestätigt und konkretisiert.

Von einem narrativen Konzept ausgehend thematisiert Viehöver (2012) Geschichten vom Klimawandel und unterscheidet sechs alternative Erzählungen, von denen drei klimaskeptisch sind: Die erste betont die positiven Folgen des Klimawandels, die sich etwa in der Verschiebung der Permafrostgrenze nach Norden zeigen. Die zweite klimaskeptische Narration normalisiert die Ursachen des Klimawandels, die danach nicht in menschgemachten Treibhausgasen liegen, sondern an natürlichen Veränderungen in der Sonnenstrahlung. Die dritte Variante schließlich handelt vom großangelegten Betrug der Öffentlichkeit durch die Wissenschaft und die Medien, die den Klimawandel als menschgemachtes Phänomen erfinden, um kommerzielle und Machtinteressen zu bedienen (Viehöver 2012, S. 203-209). Mit Viehövers Analyse decken sich zum Teil die von Brunnengräber (2013) herausgearbeiteten typischen klimaskeptischen Positionen und Argumentationsmuster. Er identifiziert fünf Aspekte, die sich in drei Bündel fassen lassen: Erstens werden im klimaskeptischen Diskurs Unsicherheiten in der wissenschaftlichen Evidenz zum Klimawandel betont. Dabei wird die methodische Grundlage, die empirischer Forschung zugrunde liegt, gegen die Klimawissenschaft eingesetzt und wasserfeste, sichere Beweise werden verlangt. Zweitens findet sich in der klimaskeptischen Argumentation die Behauptung, dass Klimawandel ein kontinuierlicher, immerwährender Prozess ist, der mit oder ohne den Menschen stattfindet. Kennzeichnend ist schließlich drittens die Rahmung des Klimawandels als Täuschung und Verschwörung, die anderen Zielen dient, etwa politischen.
Eine Publikation des Umweltbundesamts (UBA) beleuchtet vor allem die naturwissenschaftlichen Argumente der "Klimawandelskeptiker" (UBA 2013, Klappentext). Dabei werden auch zwei wissenschaftssoziologische Argumente (UBA 2013, S. 86-95) thematisiert: Der klimaskeptische Diskurs versucht den Konsens in der Wissenschaft zu destabilisieren, betont Uneinigkeit und verbreitet Falschinformation, darüber hinaus werden Klimaforsche$r$ (innen) direkt angegriffen. Diese beiden Aspekte identifiziert auch Brunnengräber (2013), der sie als Fehlinformation und "Rosinenpickerei“ (Auswahl opportuner Fakten) sowie Diffamierung bezeichnet.

Gemäß den bislang durchgeführten Studien werden im klimaskeptischen Diskurs 1. Zweifel an den Ergebnissen der Mainstreamforschung geäußert, 2. eine Täuschung der Öffentlichkeit durch die Klimaforschung unterstellt sowie 3. politische Motive aufseiten der Klimaforschung konstruiert. Diese Aspekte stehen unverbunden nebeneinander; meist liegt der Schwerpunkt der Diskussion auf den naturwissenschaftlichen Erklärungen und ihrer Widerlegung (siehe UBA 2013). Wir nehmen aber an, dass die klimaskeptische Argumentation erst durch die Anreicherung mit wissenschaftssoziologischen und politischen Argumenten einem breiteren Publikum zugänglich wird und die Beweisführung nicht nur aus den drei Komponenten bestehen kann, sondern bestehen muss. Daher möchten wir in diesem Artikel eine detaillierte Analyse klimaskeptischer Argumentation vornehmen und zeigen, wie die drei Ebenen - naturwissenschaftliche, wissenschaftssoziologische und politische - verknüpft werden und eine Narration bilden, die für Laien verständlich und plausibel erscheint und bestimmte emotionale Reaktionen nahelegt. Die vorliegende Untersuchung erweitert das Wissen über den klimaskeptischen Diskurs, indem sie 1. erstmals systematisch eine umfangreiche und klar abgegrenzte klimaskeptische Textsammlung deutsch- und englischsprachiger Sachbücher vergleichend untersucht, 2. auf dieser Grundlage die naturwissenschaftlichen, wissenschaftssoziologischen und politischen Argumente der ausgewählten Klimaskeptiker ${ }^{2}$ mit Zitaten belegt, die Argumente 3. in ihrem Zusammenhang analysiert und 4. ihre emotionale Funktion erläutert. Die Hoffnung, dass mit dem hier gebotenen Überblick auch gleich eine Fülle wirksamer Gegenstrategien erschlossen werden kann, müssen wir zwar etwas dämpfen, gleichwohl bieten wir einige praktische Schlussfolgerungen an.

\section{Klimaskeptische Sachbücher}

Das Format Sachbücher wurde gewählt, weil es sich dabei um eine besonders verbreitete Form klimaskeptischer Kommunikation handelt. Es soll mit dieser Wahl keineswegs suggeriert werden, dass klimaskeptische Ansichten nur durch dieses Medium

\footnotetext{
2 Tatsächlich sind die Verfasser der untersuchten 97 klimaskeptischen Werke bei den deutschsprachigen Sachbüchern ausschließlich, bei den englischsprachigen bis auf drei Ausnahmen (Holly Fretwell und Rosa Koire, USA,
} Donna Lafromboise, Kanada) alle männlich. 


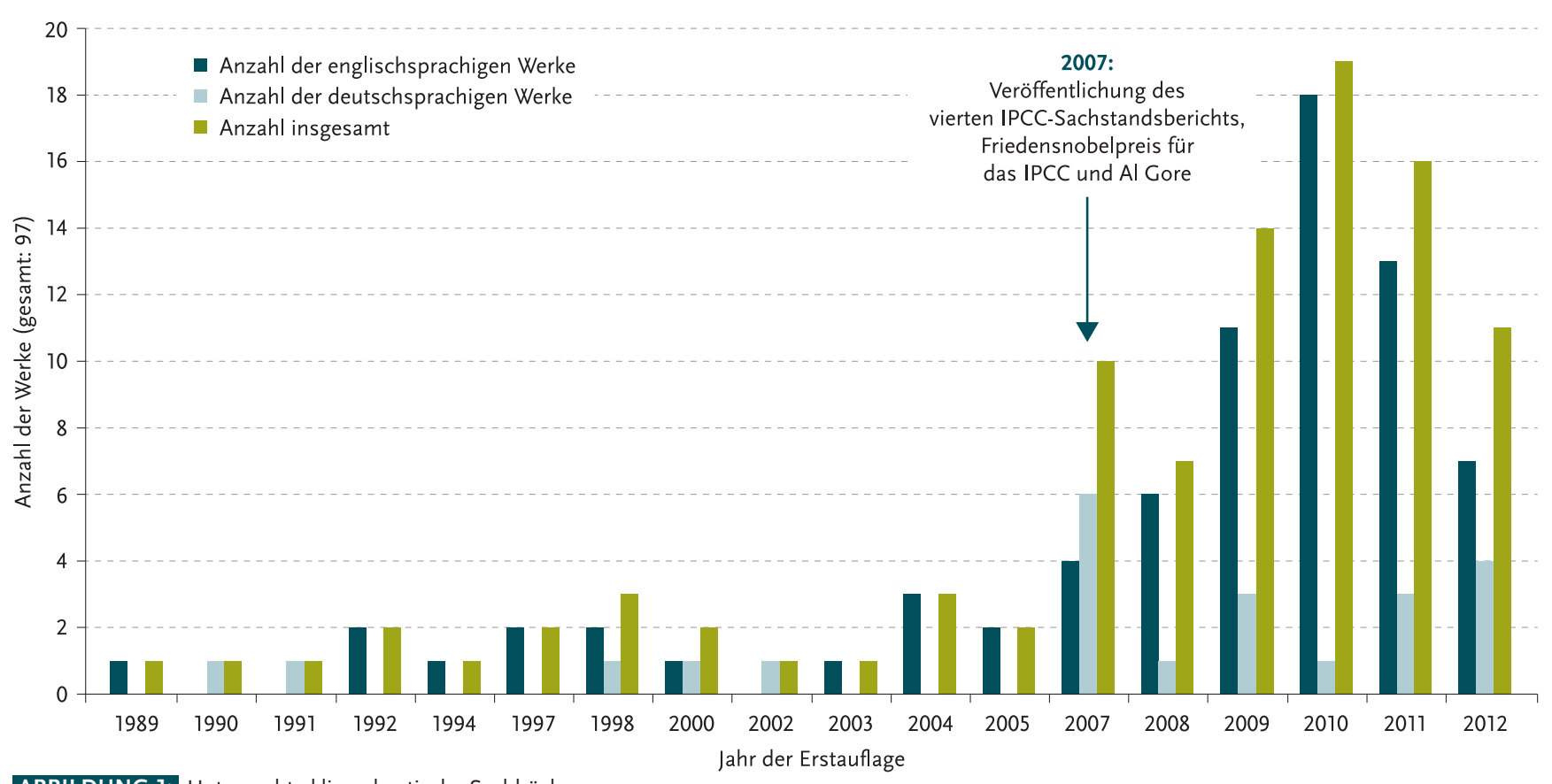

ABBILDUNG 1: Untersuchte klimaskeptische Sachbücher.

kommuniziert werden. Als Übertragungswege und Vermittlungskanäle dienen auch Kampagnen, Aufrufe, Webseiten oder Lehrbücher (siehe etwa Schultz 2000, S. 209 f.).

Sachbücher (Non-Fiction) stellen für Klimaskeptiker ein ausgesprochen beliebtes mediales Format dar, da sie eine gute $\mathrm{Zu}$ gänglichkeit ohne kleinteilige Gatekeeping-Funktion kritischer Journalist(inn)en bieten ebenso wie den nötigen Raum, um eine komplexe Materie für ein interessiertes Publikum zu behandeln. Unser Korpus umfasst nur Monografien, das heißt Werke, die sich ausschließlich oder zu mehr als einem Drittel diesem Thema widmen. Als belletristisches Werk, das eindeutig auch Sachbuchelemente aufweist und als Plädoyer für Klimaskepsis gelesen werden kann, wurde Michael Crichtons Bestseller State of Fear aufgenommen.

Der Versuch, bei den Verlagen Informationen über Auflagenzahlen zu erhalten, war nur in sehr wenigen Fällen erfolgreich. Immerhin verdeutlicht die hohe Startauflage von State of Fear mit 1,5 Millionen Exemplaren 2004, dass in bestimmten Fällen klimaskeptische Literatur weltweit ein breites Publikum erreicht.

Insgesamt konnten wir 73 englischsprachige und 24 deutschsprachige klimaskeptische Sachbücher ${ }^{3}$ identifizieren, wobei wir in unsere Untersuchung nur „starke“ klimaskeptische Bücher einbezogen haben, die die geläufige Kausaldiagnose der Klimaforschung für die globale Erwärmung ablehnen (zur Definition siehe unten). Wir haben bei den deutschsprachigen Sachbüchern ausschließlich solche gezählt, die zuerst oder zeitgleich mit der Veröffentlichung in einer anderen Sprache (zum Beispiel Tsche-

3 Alle hier untersuchten Werke sind am Wissenschaftszentrum Umwelt einsehbar, eine Liste aller analysierten Titel, die aus Platzgründen nicht aufgeführt werden können, bei der Autorin oder dem Autor erhältlich. chisch) auf Deutsch erschienen. Die Anzahl klimaskeptischer Werke war vor und nach den ersten drei Sachstandsberichten des Intergovernmental Panel on Climate Change (IPCC) (1990, 1995, 2001) zunächst gering, stieg jedoch nach dem vierten Sachstandsbericht 2007 (IPCC 2007). Im selben Jahr wurden zudem das IPCC und Al Gore mit dem Friedensnobelpreis geehrt, was den klimaskeptischen Diskurs vermutlich weiter angeregt haben dürfte (Abbildung 1).

\section{Zur Definition von Klimaskepsis}

Skepsis in Bezug auf Resultate der modernen Klimaforschung kann sich gegen folgende ihrer Annahmen richten: 1. Die globale Erwärmung ist eine Tatsache, 2. die wesentliche Ursache der globalen Erwärmung sind anthropogene Treibhausgasemissionen (vor allem $\mathrm{CO}_{2}$ ), 3. die globale Erwärmung hat für Mensch und Natur mehr negative als positive Konsequenzen, 4. Maßnahmen zur Reduktion der Emissionen sind sinnvoll, um die globale Erwärmung aufzuhalten. Klimaskepsis kann also Folgendes in Zweifel ziehen: 1. das Phänomen, 2. die Ursachendiagnose, 3. die Bewertung oder 4. die Therapie.

Bisweilen wird zu der Bezeichnung „Klimaskeptiker“ angemerkt, sie sei ungenau, da von den so Bezeichneten nicht bezweifelt werde, dass es ein Klima gebe - stattdessen sei die Bezeichnung „Klimawandelskeptiker“ (UBA 2013, Klappentext) plausibler. Allerdings ist auch diese Bezeichnung ungenau, sie müsste $\mathrm{zu}$ „Klimawandelursachendiagnosenkonsensskeptiker" erweitert werden - und auch dies wäre noch nicht hinreichend exakt. Es empfiehlt sich deshalb, bei der gebräuchlichen Bezeichnung zu bleiben. 
$\mathrm{Zu}$ beachten ist vielmehr, dass die gewöhnliche Bezeichnung „Skeptiker(in)“ zwar ihrer Wortgeschichte und ihrem Gebrauch nach nahelegt, dass jemand eine Meinung in der Schwebe lässt und unentschieden ist (griechisch sképtomai bedeutet, wie manche klimaskeptischen Personen hervorheben, „ich untersuche“). Der Glaube an Meinungen beunruhige die Seele, meinten die antiken Skeptiker, während die gleichmäßige Distanz zu allen Positionen für Seelenruhe, Gesundheit und Glück sorge (Ricken 1994, S. 101-110). Auch als wissenschaftliche Methode ist die skeptische epoché, die Zurückhaltung im Urteilen, häufig angebracht, in diesem Sinne gibt es auch in der modernen Klimaforschung zahlreiche Skeptiker(innen) (Post 2008, S. 98-103).

Doch die hier untersuchten klimaskeptischen Autoren haben mit dieser Form der Skepsis wenig zu tun. Sie sind in aller Regel nicht unentschieden, sondern entschieden, zudem entschlossen, sie haben eine Meinung und kämpfen für diese. Sie sind überzeugt, dass der übliche wissenschaftliche und politische Konsens in den genannten Punkten entweder insgesamt, mindestens jedoch in Punkt 2 sicher falsch ist. Sie sind daher keine Skeptiker im klassischen Sinn, sondern Dogmatiker, genauer: abweichende Dogmatiker (Häretiker).

Klimaskepsis im starken Sinn bestreitet mindestens die übliche Kausaldiagnose (dass menschgemachte Treibhausgase wesentliche Ursachen sind). Nur Werke, die eine solche Position vertreten, fanden Aufnahme in unseren Korpus. Wer sich hingegen in diesen Punkten dem Konsens aktueller Klimaforschung anschließt und lediglich bei Bewertung und/oder Therapie eine ablehnende oder kritische Position einnimmt, ist nur ein(e) Klimaskeptiker(in) im weiteren Sinn, wie etwa der Ökonom HansWerner Sinn oder der dänische Statistiker Björn Lomborg (nach Maßgabe ihrer Publikationen Sinn 2008 und Lomborg 2008). Ihre Argumentationen bleiben hier unberücksichtigt, ihre Werke wurden in der obigen Statistik nicht mitgezählt.

Im Folgenden betrachten wir die klimaskeptische Argumentation vom zentralen Punkt, der Kausaldiagnose, aus. Wir gehen damit von einem typischen Fall für Klimaskepsis aus, die an der Tatsächlichkeit einer (geringen) globalen Erwärmung kaum zweifelt, doch vehement den Befund der aktuellen Klimawissenschaft, globale Erwärmung sei anthropogen bedingt, bestreitet. Damit die Einwände gegen den Konsens wirken können, bedarf es, wie oben erwähnt und in Abbildung 2 veranschaulicht, zu ihrer Ergänzung einer wissenschaftssoziologischen Erklärung, die ihrerseits durch politische Thesen plausibel gemacht wird.

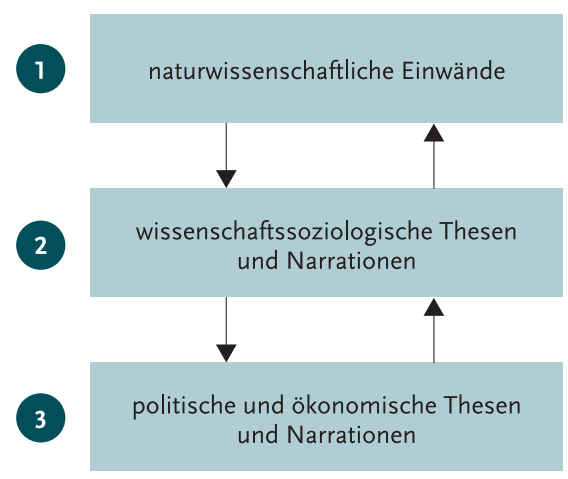

Drei Ebenen klimaskeptischer Argumentation.

\section{Die Struktur klimaskeptischer Argumentation}

\section{Einwände gegen die naturwissenschaftliche Ursachendiagnose der etablierten Klimaforschung}

Ausgangspunkt klimaskeptischer Sachbücher ist in der Regel ein mehr oder weniger tendenziöses Referat der derzeitigen Ansicht des IPCC über die Ursache der globalen Erwärmung (vergleiche Fehlinformation und „Rosinenpickerei“ bei Brunnengräber 2013). Viele der hier untersuchten Autoren versuchen, die Bedeutung der minimalen $\mathrm{CO}_{2}$-Menge in der Atmosphäre herunterzuspielen, weil eine so winzige Quantität keine Effekte haben könne (siehe Krüger 2012, S. 27, Horowitz und Laksin 2012, S. 131). Sie dürften außerhalb wissenschaftlich diskutierbarer Positionen stehen; denn dass $\mathrm{CO}_{2}$ trotz seines geringen prozentualen Anteils in der Atmosphäre durchaus enorme Relevanz für natürliche Prozesse hat, zeigt schon die Existenz von Pflanzen, die sich vor allem von $\mathrm{CO}_{2}$ ernähren.

Doch dass die $\mathrm{CO}_{2}$-Konzentration der Luft prinzipiell ein klimawirksamer Faktor sein kann, wird selten bestritten. Bevorzugt werden Fälle von Klimawandel betrachtet, in denen $\mathrm{CO}_{2}$ nicht maßgeblich war; daraus wird geschlossen, dass es auch für die beobachtete globale Erwärmung keine Rolle spiele. Dies ist kein gültiger Schluss, da das Klimasystem zum einen von mehreren Faktoren angetrieben wird, zum anderen hemmende Faktoren die Wirkung des $\mathrm{CO}_{2}$ aufheben können. In der Tradition der guten Rhetorik folgen die Klimaskeptiker der Empfehlung von Aristoteles, Beispiele und keine umständlichen Induktionsschlüsse zu präsentieren (Aristoteles 2002, S. 1356 b). Meist wird anhand des (von der Klimageschichte zum Teil bestrittenen) mittelalterlichen Wärmeoptimums gezeigt, dass Erwärmungen auch ohne Erhöhung des $\mathrm{CO}_{2}$-Spiegels der Luft möglich sind. Ebenso wird gern die Stagnation der globalen Erwärmung seit etwa 1998 als Beleg angeführt: „Once again, science is the enemy of alarmists. Atmospheric $\mathrm{CO}_{2}$ has continued to slowly increase since 1980, but global warming has stopped. There has been no warming for ten years, since 1998, and the entire increase in global temperature over the last century was wiped out in the last two years. How can this be? The answer is that Earth's climate has always been governed by natural causes, not $\mathrm{CO}_{2}$ “ (Johnson 2009, S. 53).

Aus der gleichbleibenden durchschnittlichen Erdtemperatur trotz steigenden $\mathrm{CO}_{2}$-Gehalts in der Atmosphäre wird auf die kausale Irrelevanz des $\mathrm{CO}_{2}$ für das Klima und auf den größeren Einfluss natürlicher Ursachen geschlossen und es wird der „eigentlich“ relevante Faktor präsentiert. Dies ist in aller Regel die Sonne, es wird aber ebenso die natürliche Variabilität des Klimasystems als Ursache von Klimaänderungen geltend gemacht. Belegt wird die Behauptung gelegentlich mit Daten, häufiger aber auch nur mit Verweis auf die Größe der Sonne - im Vergleich zu den winzigen Mengen $\mathrm{CO}_{2}$ im Klimasystem. Die Betonung natürlicher Ursachen für die Klimavariabilität schließt den naturwissenschaftlichen Teil klimaskeptischer Argumentation im Wesentlichen ab. Aus Sicht der etablierten Klimaforschung stehen die Argumente auf schwachen Füßen - zu jedem weiß sie eine
Erwiderung (vergleiche dazu etwa die Publikation des UBA 2013). 


\section{Wissenschaftssoziologische These: Verschwörung der Klimaforschung}

Unabhängig davon, ob die naturwissenschaftlichen klimaskeptischen Argumente überzeugen oder nicht, drängt sich folgender Einwand auf: Wenn das menschgemachte Treibhausgas nicht Ursache der aktuellen Klimaerwärmung ist, sondern die Sonne oder die natürliche Variabilität des Klimasystems (oder andere Faktoren), warum spricht dann die aktuelle Klimaforschung (und überhaupt die ganze Welt) so viel vom $\mathrm{CO}_{2}$ ? Wie kann dann der vierte Sachstandsbericht des IPCC (2007) als Konsens erklären, anthropogene Treibhausgase, vor allem $\mathrm{CO}_{2}$, seien sehr wahrscheinlich die Ursache der globalen Erwärmung?

Es gibt klimaskeptische Werke, die zu dieser Frage schweigen (zum Beispiel Berner 2000). 72 von 97 Titeln jedoch, also nahezu 75 Prozent der klimaskeptischen Autoren, lösen das Problem, indem sie die Argumentation erweitern durch die Formulierung wissenschaftssoziologischer Thesen, die von politischen Annahmen flankiert werden. Eine solche Erweiterung oder auch Eskalation hat zum einen die Funktion, die skeptische Rückfrage zu beantworten, wie es denn sein könne, dass die Mehrheit der etablierten Klimawissenschaftler(innen) anderes verkündet als die klimaskeptischen Personen. Sie hat aber zum anderen auch eine klar emotionalisierende Funktion - durch die Schaffung eines Feindbilds.

Wissenschaftstheoretisch wird gegen die moderne Klimawissenschaft zunächst oft ins Feld geführt, dass Konsens kein Kriterium für Wahrheit sei, die aktuelle Meinung der Klimaforschung aber auf weiter nichts als einem Konsens beruhe - möglicherweise sogar nur ein "gefälschter“, gewaltsam „geschmiedeter“ Konsens sei, wie der Klimaskeptiker Lindzen (1996) behauptet (ähnlich dazu auch Crichton 2005, S. 684). Wissenschaftssoziologisch wird dann erläutert, wie sich dieser Konsens durchsetzen konnte. Hier gelangen wir zu einem oft narrativ ausgeschmückten, wenn auch bislang wenig beachteten Teil klimaskeptischer Argumentation, der die wichtige Funktion hat zu erläutern, weshalb trotz der von skeptischen Lagern präsentierten „Sachlage“ weltweit vom Klimawandel und vom $\mathrm{CO}_{2}$ gesprochen wird und Aktivitäten zur Bekämpfung der vermeintlichen Ursachen des Klimawandels stattfinden. Diese intuitive Wissenschaftssoziologie schließt kaum und, wie weiter unten gezeigt wird, nur oberflächlich an den akademischen Diskurs der Wissenschaftssoziologie an. Ihre Funktion ist eine rhetorische - sie will nicht zur Forschung beitragen, sondern sie will ein emotionalisiertes Bild von Wissenschaft entwerfen, das die eigene Argumentation stärkt und ergänzt.

Als Erklärung wird meist eine Variante von Verschwörungstheorie präsentiert, die die Täuschung der Öffentlichkeit durch die Klimawissenschaft und Verbündete in der Politik annimmt. Diese Täuschung kann aber, weil die Klimaforschung ein soziales Unternehmen ist, nur als eine kollektive funktionieren, nämlich als Zusammenschluss mehrerer Personen mit dem Zweck, unter Umgehung geltender ethischer und/oder rechtlicher Standards und mit Hilfe gezielter Irreführung ein gemeinsames Ziel zu erreichen.
$\mathrm{Zu}$ Verschwörungstheorien gibt es eine reichhaltige Literatur. Danach handelt es sich um diskursive Konstruktionen, „to explain some event or practice by reference to the machinations of powerful people, who attempt to conceal their role (at least until their aims are accomplished)“ (Sunstein und Vermeule 2009, S. 205, siehe auch Grant 2011). Barkun (2003) identifiziert drei Aspekte, die allen Verschwörungstheorien gemein sind: die Überzeugung, dass die Zustände der Welt kein Zufall sind, sondern auf intentionale Akte zurückzuführen, dass der Anschein trügen kann und scheinbar harmlose Ereignisse oder Gruppen in Wirklichkeit schädlich sind und dass alles zusammenhängt - auch nach außen hin unverbundene Sachverhalte können eine geheime Verbindung haben, die man bei einer aufmerksamen Suche nach Mustern entdecken kann. Verschwörungstheorien sind nicht falsifizierbar, sie sind vielmehr ausreichend flexibel, um Gegenargumente und widersprechende Fakten passend zur eigenen Position umzudeuten (Kuhn 2010).

„Der typische diskursive Nutzen einer Darstellung unliebsamer Sachverhalte als intendierte Effekte geheimer Organisation liegt (...) gerade darin, dass man diese Sachverhalte als abstellbar darstellt und die Methode ihrer Abstellung gleich mitliefert, nämlich durch Abschaffung der Organisation“ (Kuhn 2010, S. 120). Eine bedrohliche Situation wie der Klimawandel, der auch bei maximalen Bemühungen nicht vollständig durch Menschen kontrollierbar ist und erhebliche Folgen für die Menschen haben kann, wird in eine andere, weitaus weniger bedrohliche Situation uminterpretiert, nämlich die, in der eine überschaubare Gruppe von Menschen zu ihrem eigenen Vorteil konspiriert; eine Angelegenheit, die zutiefst menschlich und mit gewöhnlichen menschlichen Mitteln zu regeln ist. Verschwörungstheorien um den Klimawandel haben also eine besondere Anziehungskraft, weil sie die Folgen des Klimawandels weniger gravierend erscheinen lassen und damit entlasten. Sie sind insoweit Antworten auf apokalyptische Narrationen von Klimaengagierten, die zwar nicht in der eigentlich wissenschaftlichen Literatur, wohl aber in der öffentlichen Berichterstattung über die Klimaforschung verbreitet sind (Weingart et al. 2008). Zugleich wirken sie emotional, weil sie Gefühle wie Sorge und Angst vor einer möglicherweise nicht mehr abwendbaren Katastrophe ersetzen durch herzhaften Zorn auf eine Menschengruppe, die andere an der Nase herumführt.

Viele Klimaskeptiker sehen sich daher als Aufklärer; bisweilen wird gar der Evangelist Johannes zitiert, um die Dignität der Enthüllungen zu unterstreichen: „And the truth, as always, really will set you free. Congratulations. You've taken the first step towards reclaiming your freedom from fear. Don't let it be the last such step you take“ (Wishart 2009, S. 272). Offensichtlich fällt das klimaskeptische empowerment anders aus, als die Klimaengagierten sich dies wünschen (vergleiche zu klimaengagiertem empowerment Leggewie und Welzer 2009, S. 196 f.) (Abbildung 3). Der klimaskeptische Diskurs will seine Leser(innen) befreien, sie sind nach Lektüre und Aufnahme der Botschaft nicht mehr ängstliche Empfänger(innen) der Warnungen anderer, sondern können darangehen, selbst andere zu warnen, nämlich vor der nun „aufgedeckten“ Verschwörung. 
Diese emotionalen Vorzüge vermögen den entscheidenden sozialen Nachteil der klimaskeptischen Literatur, dass sie sich nämlich gegen den wissenschaftlichen und gesellschaftlichen Mainstream stellt, zumindest für manche Leser(innen) aufzuwiegen. Dass die Emotionalisierung gezielt gewollt ist, belegen die Argumentationsalternativen, die durchaus auch zur Verfügung stünden: Man könnte auch argumentieren, dass die Klimaforscher(innen) sich einfach irren oder sich in ihrer Hypothese gleichsam festgebissen hätten und alle widersprechenden Fakten als Anomalien verdrängten. Eine solche Erklärung, die auf moderne wissenschaftstheoretischen Auffassungen wie Thomas Kuhns Paradigmentheorie zurückgreift, stellte die Klimaforscher(innen) gewissermaßen als betrogene Betrüger(innen) dar, die in ihrem Paradigma gefangen sind. Einzelne klimaskeptische Autoren zitieren Kuhn (Michaels und Balling 2005, S. 191-198, MacRae 2010, S. 130-150). Sehr weit von der Verschwörungstheorie ist aber auch diese gelehrte Variante der Klimaskepsis nicht. Mit Versatzstücken aus der Public-Choice-Theorie wird nämlich das Kuhn'sche Problem der Theoriewahl derart gelöst, dass in der Klimaforschung diejenigen Theorien gewählt werden, die finanzielle Vorteile bieten.

Neutrale Erklärungen, weshalb sich die Klimaforschung aus skeptischer Sicht auf dem Abweg befindet, sind kaum aufzufinden, wenig überraschend angesichts des rhetorischen Nachteils einer solchen Argumentation. Sie liefert kein Feindbild, auf das man wütend sein kann. Zorn kann nicht entstehen, wenn jemand präsentiert wird, die oder der unabsichtlich täuscht, weil sie oder er selbst im Irrtum befangen ist. So entstünde vielmehr Nachsicht oder Mitleid. Zorn kann entstehen, wenn jemand gezeigt wird, die oder der die Wahrheit kennt, diese aber aus niederen Motiven verbirgt (siehe bereits Aristoteles 2002, 1378b-1380 b). Wollen also Klimaskeptiker(innen) ihre Mitmenschen mit einer empörenden Entdeckung wachrütteln und mobilisieren und ihrer aufklärerischen Mission treu bleiben, muss die Verschwörungstheorie attraktiver als andere Argumentationsalternativen sein. Und das ist sie in der Tat, wie unsere Auswertung zeigt.

\section{Politische Ziele der unterstellten Verschwörung: Eigennutz und Gesellschaftsumbau}

Welche Ziele verfolgt die Verschwörung? Normalerweise werden Geld und Ansehen genannt: „Eine Überspitzung der vermeintlichen Klimagefahr lässt die staatlichen Forschungsgelder weiter üppig sprudeln, führt zur Neugründung von Instituten und der Aufstockung der Wissenschaftlerstellen. Die postulierte Klimagefahr schafft Karrieremöglichkeiten, Prestige, Medienbühne und politische Beraterrollen für vormals unbeachtete Klimaforscher" (Vahrenholt und Lüning 2012, S. 274).

Neben Geld und Ansehen gibt es weitere Motive, die politisch sind. Die Klimaengagierten möchten - aus Sicht der klimaskeptischen Position - die Gesellschaft ändern. So beobachten Vahrenholt und Lüning (2012, S. 326) in der Klimadiskussion eine „steigende Tendenz, es nicht nur bei (...) moralischen Appellen zu belassen, sondern über Gesetze und Verordnungen direkt in das Leben der Bürger einzugreifen“. Und genau dies führt nach

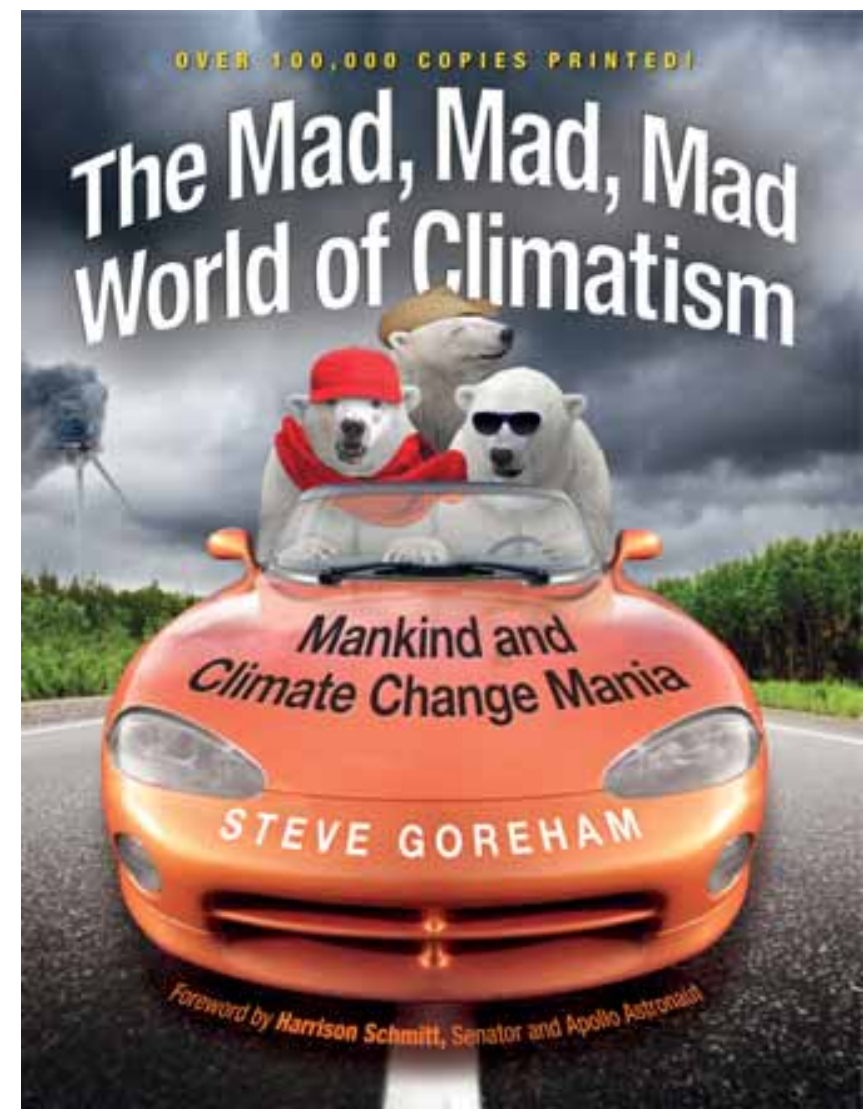

ABBILDUNG 3: Die Eisbären sind los! Die „verkehrte Welt“ der Klimaengagierten wird auf diesem Cover eines klimaskeptischen Werks sarkastisch karikiert.

Ansicht der Klimaskeptiker auf einen linken Weg des Unheils. Es handele sich um „utopischen Klima-Jakobinismus“ (Vahrenholt und Lüning 2012, S. 330). Klimaskeptische Personen in den Vereinigten Staaten malen die Gefahr eines linken Umsturzes mit grüner Camouflage drastisch aus. Zwar wird nicht oft so explizit formuliert wie beim konservativen US-amerikanischen Journalisten Brian Sussman, der sein Buch Climategate: A Veteran Meteorologist Exposes the Global Warming Scam mit dem Satz beginnt: „Global Warming's story begins with a diabolical bastard named Karl Marx“ (Sussman 2010, S. IX). Aber es wird häufig ein Bezug zwischen Umweltbewegung und Sozialismus hergestellt und die antikapitalistische und freiheitsbegrenzende Stoßrichtung unterstrichen. Christopher Horner argumentiert eleganter, in der Sache aber ähnlich: „This is not your father's environmental movement. Your hippie uncle certainly wouldn't recognize it. (...) Most importantly for you: environmental causes always include - and often are primarily - campaigns to gain more government control over the economy and individual activity. They are never fights for less control or greater liberty. When communism didn't work out, environmentalism became the anti-capitalist vehicle of choice, drawing cash and adoration from business, Hollywood, media, and social elites. Environmental pressure groups have boomed into a 2 billion dollar industry“ (Horner 2007, S. 3). 
Ein weiteres Motiv, das von den Klimaskeptikern ins Spiel gebracht wird, sind neokolonialistische Interessen. Die Klimaengagierten haben angeblich das Ziel, Länder des Südens in wirtschaftlicher Unterentwicklung zu halten, um selbst Profite zu machen: „(A)t a time when fully industrialized nations find themselves at a massive advantage in the world of economic competition, calls for cap-and-trade should be heard for what they are: a demand for a lengthy - if not permanent - time-out in the development game. (...) Al Gore's moral army has taken a position that serves the elite of the best-off countries in the world“ (Ambler 2011, S. 38f.). Auch deutschsprachige Klimaskeptiker sprechen dieses Motiv an: „Die Atmosphäre wird dadurch sicher nicht sauber, doch Rückständigkeit und Unterentwicklung werden durch eine derartige Energiepolitik zementiert" (Schauerhammer 1990, S. 23, ähnlich Driessen 2006, S. 135).

\section{Angesichts steigender individueller Klimawandelkosten könnte die Offenheit für klimaskeptische Positionen in der Öffentlichkeit wachsen.}

Die wichtigsten von Klimaskeptikern unterstellten Motive sind: 1. Die Klimawissenschaft hat sich verschworen, um Macht zu gewinnen und/oder Forschungsgelder zu sichern, 2. die Klimawissenschaft hat sich verschworen, um unter grünem Deckmantel Gesellschaftsumbau oder -umsturz zu betreiben (meist sozialistisch oder kommunistisch inspiriert), 3. die Klimawissenschaftler(innen) handeln im Dienst einer Verschwörung ehemaliger Kolonialmächte, die frühere Kolonien und jetzige Industrienationen wie Indien oder Brasilien über den Umweg etwa von Emissionsminderungszielen am weiteren Wachstum hindern wollen (siehe auch Bachram 2004).

\section{Was tun mit den Klimaskeptikern?}

Bei Klimaengagierten ruft die Konfrontation mit klimaskeptischer Literatur meist Unwillen oder gar Verachtung hervor. Deren wissenschaftliche Argumente seien schon Dutzende Male widerlegt worden. Der Widerwille ist nachvollziehbar. Wir decken mit unserer Analyse der klimaskeptischen Argumentation den Zusammenhang zwischen naturwissenschaftlichen, wissenschaftssoziologischen und politisch-ökonomischen Argumenten auf. Gezeigt wurde, dass gerade die intuitive Wissenschaftssoziologie der Klimaskepsis und ihre politischen Thesen die Emotionalisierung tragen. Wie angreifbar diese Elemente der klimaskeptischen Argumentation sind, bedarf kaum des Hinweises.

Die sachliche Auseinandersetzung wird erschwert, wenn die Emotionalisierungsstrategie greift - Letztere als solche offenzulegen dürfte daher nützlich sein. Weniger empfehlenswert ist da- gegen, mit einer Gegenemotionalisierung zu antworten, wie es unter anderem das UBA für richtig hält, indem nun umgekehrt eine von den klimaskeptischen Lagern ins Werk gesetzte, von der Ölindustrie finanzierte Verschwörung suggeriert wird (UBA 2013, S. 100-105, Brunnengräber 2013, S. 42 und passim, der unter anderem die Journalisten Dirk Maxeiner und Michael Miersch als „Industrielobbyisten“ brandmarkt, siehe auch Blawsberg und Kohlenberg 2012). Es ist wenig sinnvoll, allen klimaskeptischen Personen von vornherein niedere (finanzielle) Motive zu unterstellen. Zwar gibt es bezahlte Klimaskeptiker(innen) in den Vereinigten Staaten (Oreskes und Conway 2010); dort unterhalten zudem zahlreiche Klimaskeptiker(innen) enge Beziehungen zu konservativen Thinktanks (Jacques et al. 2008). Daneben gibt es aber auch Autor(inn)en, die ihre Position aus ehrlicher Überzeugung vertreten und für ihre vom Mainstream abweichende Meinung nicht nur keine Belohnungen kassieren, sondern womöglich soziale Nachteile in Kauf nehmen.

Insgesamt scheint die Polarisierung zwischen den Klimaskeptiker(inne)n und den Klimaengagierten so weit fortgeschritten, dass auf argumentativem Weg Angehörige des einen Lagers nicht mehr ins andere wechseln werden und umgekehrt. Beleg für die verhärteten Fronten ist der Fall eines konvertierten Klimaskeptikers, des amerikanischen Physikers Richard Muller (Berkeley), der weltweit in den Medien als Sensation kommentiert wurde.

In dieser Situation wird vor allem die Entwicklung des Klimas selbst die Basis für individuelle und kollektive Sichtweisen sein, denn wenn die weltweiten Durchschnittstemperaturen in der nächsten Dekade trotz kontinuierlich steigender $\mathrm{CO}_{2}$-Emissionen stabil bleiben oder gar fallen, dürfte die Offenheit für klimaskeptische Positionen wachsen. Da zudem die Haltung zum Klimawandel möglicherweise stärker von sozialen, ökonomischen und politischen Aspekten bestimmt wird als von naturwissenschaftlichen Fakten, könnte mit steigenden individuellen Kosten der Energiewende (höhere Energiepreise, Veränderung der Landschaft durch Windräder und Solarstromanlagen etc.) die Offenheit gegenüber klimaskeptischen Positionen - auch im umweltfreundlichen Deutschland - zusätzlich steigen.

Wir danken Cornelia Schnell, Andrea Heigl und Julia Fendt für Unterstützung bei der Auswertung der klimaskeptischen Literatur, der Universitätsbibliothek Augsburg, insbesondere Gabriele Bihler, für Hilfe bei der Beschaffung der Literatur sowie den anonymen Gutachter(inne)n und der Hauptherausgeberin für konstruktive Kritik an früheren Versionen dieses Textes.

\section{Literatur}

Ambler, H. 2011. Don't sell your coat: Surprising truths about climate change. East Greenwich, RI: Lansing International.

Antilla, L. 2005. Climate of scepticism: US newspaper coverage of the science of climate change. Global Environmental Change - Human and Policy Dimensions 15/4: 338-352.

Aristoteles. 2002. Rhetorik. In: Werke. Übersetzt und erläutert von Christof Rapp. Bd. 4, 1. Hlbbd. Darmstadt: Wissenschaftliche Buchgesellschaft.

Bachram, H. 2004. Climate fraud and carbon colonialism: The new trade in greenhouse gases. Capitalism Nature Socialism 15: 1-16.

Barkun, M. 2003. A culture of conspiracy. Berkeley, CA: University of California Press. 
Berner, U. 2000. Klimafakten: Der Rückblick, ein Schlüssel für die Zukunft. Stuttgart: Schweizerbart.

Blawsberg, A., K. Kohlenberg. 2012. Die Klimakrieger. Zeit Dossier. Die Zeit 48, 22.11.2012: 17-19.

Brunnengräber, A. 2013. Klimaskeptiker in Deutschland und ihr Kampf gegen die Energiewende. IPW Working Paper 1/2013. Universität Wien: Institut für Politikwissenschaft. http://politikwissenschaft.univie.ac.at/fileadmin/ user_upload/inst_politikwiss/IPW_Working_Papers/IPW-WP-1_2013Achim-Brunnengraeber.pdf (abgerufen 21.06.2013).

Crichton, M. 2005. State of fear. London: Harper Collins.

Driessen, P. 2006. Öko-Imperialismus: Grüne Politik mit tödlichen Folgen. Jena: TvR Medienverlag.

Gavin, N.T., T. Marshall. 2011. Mediated climate change in Britain: Scepticism on the web and on television around Copenhagen. Global Environmental Change - Human and Policy Dimensions 21/3: 1035-1044.

Goreham, S. 2012. The mad, mad, mad world of climatism. New Lenox, IL: New Lenox Books.

Grant, J. 2011. Denying science: Conspiracy theories, media distortions, and the war against reality. Amherst, NY: Prometheus Books.

Hoggan, J. 2009. Climate cover-up: The crusade to deny global warming. Vancouver, BC: Greystone Books.

Horner, C. C. 2007. The politically incorrect guide to global warming and environmentalism. Washington, D. C.: Regnery.

Horowitz, D., J. Laksin. 2012. The new Leviathan: How the left-wing money machine shapes American politics and threatens America's future. New York: Crown Forum.

Hulme, M. 2009. Why we disagree about climate change: Understanding controversy, inaction and opportunity. Cambridge, UK: Cambridge University Press.

IPCC (Intergovernmental Panel on Climate Change). 1990. First assessment report. Geneva: IPCC. www.ipcc.ch/ipccreports/1992\%20IPCC\%20 Supplement/IPCC_1990_and_1992_Assessments/English/ipcc_90_92_ assessments_far_overview.pdf (abgerufen 26.02.2014).

IPCC. 1995. Second assessment climate change 1995. A report of the Intergovernmental Panel on Climate Change. Genf: IPCC.

IPCC. 2001. Climate change 2001: Synthesis report. A contribution of working groups I, II, and III to the third assessment report of the Intergovernmental Panel on Climate Change. Cambridge, UK: Cambridge University Press.

IPCC. 2007. Climate change 2007: Synthesis report. A contribution of working groups I, II and III to the fourth assessment report of the Intergovernmental Panel on Climate Change. Genf: IPCC.

Jacques, P.J., R. E. Dunlap, M. Freeman. 2008. The organisation of denial: Conservative think tanks and environmental scepticism. Environmental Politics 17/3: 349-385.

Johnson, L. 2009. Understanding the global warming hoax, expanded and updated. Oakland, OR: Red Anvil Press.

Kerr, R. A. 2009. Amid worrisome signs of warming, "climate fatigue" sets in. Science 326/5955: 926-928.

Krüger, G. 2012. Die Energiewende: Wunsch und Wirklichkeit. Norderstedt: Books on Demand.

Kuhn, O. 2010. Spekulative Kommunikation und ihre Stigmatisierung am Beispiel der Verschwörungstheorien. Ein Beitrag zur Soziologie des Nichtwissens. Zeitschrift für Soziologie 39: 106-123.

Leggewie, C., H. Welzer. 2009. Das Ende der Welt, wie wir sie kannten: Klima, Zukunft und die Chancen der Demokratie. Frankfurt am Main: Fischer.

Leuschner, A. 2012. Die Glaubwürdigkeit der Wissenschaft: Eine wissenschaftsund erkenntnistheoretische Analyse am Beispiel der Klimaforschung. Bielefeld: transcript.

Lindzen, R. 1996. Global warming and eugenics. In: From risks, costs, and lives saved. Herausgegeben von R. W. Hahn. New York: Oxford University Press. 85-103.

Lomborg, B. 2008 (orig. 2007). Cool it! Warum wir trotz Klimawandels einen kühlen Kopf bewahren sollten. München: Deutsche Verlagsanstalt.

MacRae, P. 2010. False alarm: Global warming. Facts versus fears. Victoria, BC: Spring Bay Press.

Michaels, P.J., Balling, R. C., Jr. 2005. The Satanic gases: Clearing the air about global warming. Washington, D. C.: Cato Institute.

Nicholson, F. 2010. The politics of climate (dis) belief. Nature 468/7324: 634.
Oreskes, N., E. Conway. 2010. Merchants of doubt: How a handful of scientists obscured the truth on issues from tobacco smoke to global warming. New York: Bloomsbury.

Poortinga, W., A. Spence, L. Whitmarsh, S. Capstick, N. F. Pidgeon. 2011. Uncertain climate: An investigation into public scepticism about anthropogenic climate change. Global Environmental Change Human and Policy Dimensions 21/3: 1015-1024.

Post, S. 2008. Klimakatastrophe oder Katastrophenklima? Die Berichterstattung über den Klimawandel aus Sicht der Klimaforscher. Reihe medien Skripten 51. München: Reinhard Fischer.

Ricken, F. 1994. Antike Skeptiker. München: C. H. Beck.

Schauerhammer, R. 1990. Sackgasse Ökostaat: Kein Platz für Menschen. Wiesbaden: Dr. Böttiger.

Schultz, J. 2000. Handbuch der Ökozonen. Stuttgart: Ulmer.

Sinn, H.-W. 2008. Das grüne Paradoxon: Plädoyer für eine illusionsfreie Klimapolitik. Berlin: Econ.

Sunstein, C. R., A. Vermeule. 2009. Conspiracy theories: Causes and cures. Journal of Political Philosophy 17/2: 202-227.

Sussman, B. 2010. Climategate: A veteran meteorologist exposes the global warming scam. Washington, D. C.: WND Books.

UBA (Umweltbundesamt) (Hrsg.). 2013. Und sie erwärmt sich doch. DessauRoßlau: UBA. www.umweltbundesamt.de/sites/default/files/medien/ 378/publikationen/und_sie_erwaermt_sich_doch_131201.pdf (abgerufen 14.02.2014).

Vahrenholt, F., S. Lüning. 2012. Die kalte Sonne: Warum die Klimakatastrophe nicht stattfindet. Hamburg: Hoffmann und Campe.

Viehöver, W. 2012. Öffentliche Erzählungen und der globale Wandel des Klimas. In: Erzählungen im Öffentlichen. Herausgegeben von M. Arnold et al. Wiesbaden: VS Verlag für Sozialwissenschaften. 173-215.

Weart, S. 2011. Global warming: How skepticism became denial. Bulletin of the Atomic Scientists 67/1: 41-50.

Weingart, P., A. Engels, P. Pansegrau. 2008. Von der Hypothese zur Katastrophe: Der anthropogene Klimawandel im Diskurs zwischen Wissenschaft, Politik und Massenmedien. Opladen: Barbara Budrich.

Whitmarsh, L. 2011. Scepticism and uncertainty about climate change: Dimensions, determinants and change over time. Global Environmental Change - Human and Policy Dimensions 21/2: 690-700.

Wishart, I. 2009. Air con: The seriously inconvenient truth about global warming. Auckland, NZ: Howling at the Moon.

Eingegangen am 8. Juli 2013; überarbeitete Fassung angenommen am 18. November 2013.

Jens Soentgen

Geboren 1967 in Bensberg, Nordrhein-Westfalen. Studium der Chemie, Soziologie und Philosophie. Promotion 1997. 1999 bis 2001 mehrfach Gastdozent für Philosophie in Brasilien. Seit 2002 wissenschaftlicher Leiter des Wissenschaftszentrums Umwelt der Universität Augsburg.

Seit 2012 Mitherausgeber von GAIA. Entwicklung von Ausstellungen über Staub, $\mathrm{CO}_{2}$ und Stickstoff. Forschungsschwerpunkte: science and technology studies, Wissenschaftskommunikation, Stoffgeschichten.

\section{Helena Bilandzic}

Geboren 1972 in München. Studium der Kommunikationswissenschaft, Romanistik und des Medienrechts.

2003 Promotion, 2009 Habilitation. Seit 2010 Professorin für Kommunikationswissenschaft mit Schwerpunkt Rezeption und Wirkung an der Universität Augsburg.

Forschungsschwerpunkte: Rezeptions- und Wirkungsforschung, insbesondere Verarbeitung, Erleben und Wirkung narrativer Medienangebote, Mediennutzung, Methoden und Methodologie, Umweltkommunikation. 